\title{
Design of peptidic inhibitors targeting the dimerization interface of galectins
}

\author{
Jacinthe Gagnon ${ }^{1}$, Philippe Egesborg ${ }^{1}$, Maria Claudia Vladoiu ${ }^{1}$, Yves St-Pierre ${ }^{1}$, David Chatenet ${ }^{1}$, Nicolas Doucet ${ }^{1}$ \\ ${ }^{1}$ INRS - Institut Armand-Frappier, Laval, Canada \\ E-mail: jacinthe.gagnon@iaf.inrs.ca
}

Galectins are small soluble lectins that bind to galactosides via their carbohydrate recognition domain (CRD). They are involved in a variety of biological functions such as cell adhesion and recognition of pathogens. They are also known to modulate cell migration, metastasis and survival. Because their dysregulation is often observed in cancer cells, they are interesting modulators of tumor progression and attractive targets for cancer therapy. Most galectin inhibitors developed so far target the CRD. However, since the CRD is very conserved between the galectins, these inhibitors normally lack specificity and are ineffective against CRD-independent functions. [1,2]

Galectin-7 is a prototype galectin that attracts increasing interest due to its implication in various cancers. Galectin-7 ability to form homodimers has been demonstrated essential for its ability to promote tumor formation and progression. The dimer formation in galectin-7 occurs in a "back-to-back" structure (Figure 1).[1,3]

In this study, peptides corresponding to dimer interface segments were synthesized with or without the addition of a polyethylene glycol chain at the $\mathrm{N}$-terminus to improve solubility. The binding of the peptides to galectin-7 was evaluated along with their ability to disrupt galectin-7 dimer formation and to modulate associated biological activities. In parallel, other residues having a role in the dimer stabilization were identified using PyMOL software. Residues R14, R20 and D94 at the dimerization interface were selected and alanine mutants at these positions were produced and purified. Evaluation of the impact of these substitutions on structure, in absence and in presence of ligands, galectin-7 dimer formation as well as affinity to CRD ligands such as lactose, galactose and LacNAc was undertaken.

This study will probably highlight key features in the dimerization of galectin-7 and potential targets for the development of galectin-7 specific inhibitors not altering CRD functions or other galectin activities.

Figure 1: A) Structure of galectin-7 dimer (PDB ID 1BKZ). The residues 129-135 in the dimerization interface are colored in green and purple. B) Structural representation of the residues 129-135 in the first galectin-7 monomer (various colors) interacting with those in the second monomer (black). Hydrogen bonds and electrostatics interactions are indicated by dashed lines. These images were prepared by PyMOL.[3]

[1] Vladoiu, M. C. et al. (2014). Int. J. Oncol., 44, 1001-1014.

[2] Liu, F. T. \& Rabinovich, G. A. (2005). Nat. Rev. Cancer, 5, 29-41.

[3] Vladoiu, M. C. et al. (2015). Oncotarget, 6, 40970-40980.

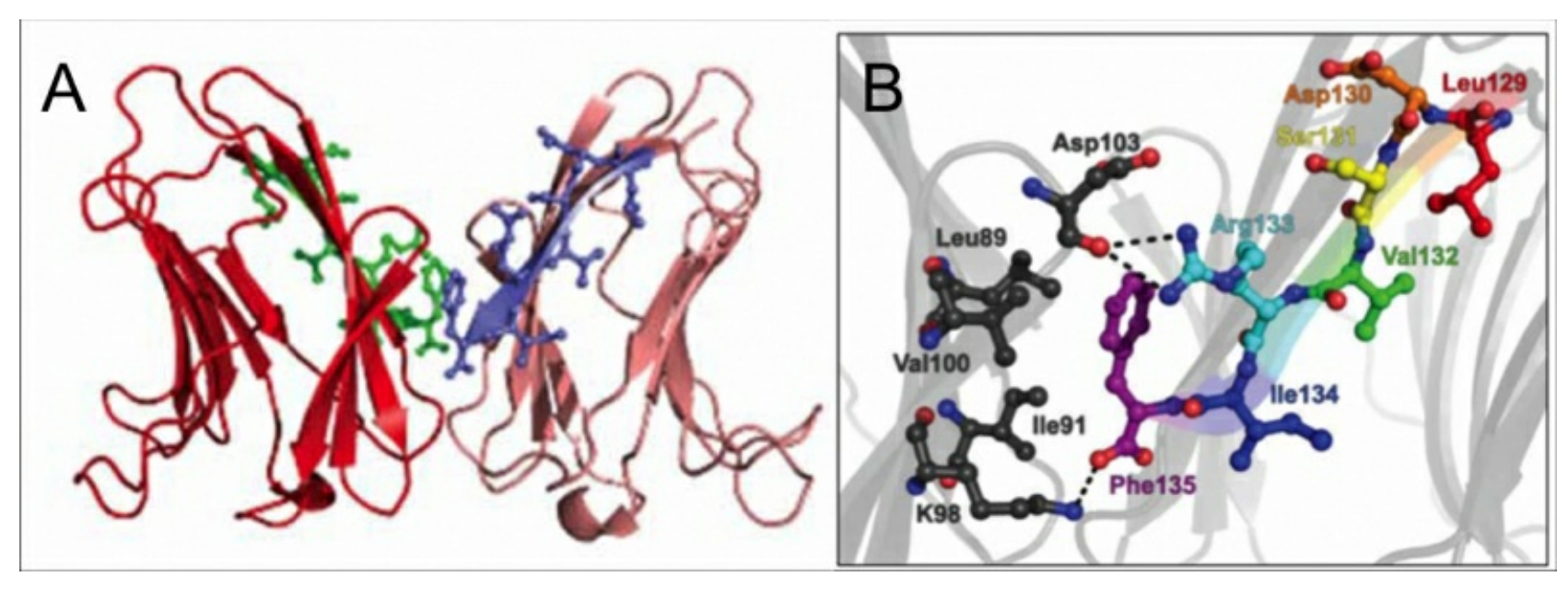

Keywords: Galectins, inhibitors, dimerization 